\title{
Quantum Statistics in Physical Chemistry, the Law of Mass Action and Epicatalysis
}

\author{
George S. Levy \\ Entropic Power, Irvine, CA, USA \\ Email: glevy@entropicpower.com
}

How to cite this paper: Levy, G.S. (2018) Quantum Statistics in Physical Chemistry, the Law of Mass Action and Epicatalysis. Open Journal of Physical Chemistry, 8, $81-99$.

https://doi.org/10.4236/ojpc.2018.84006

Received: October 12, 2018

Accepted: November 17, 2018

Published: November 20, 2018

Copyright $\odot 2018$ by author and Scientific Research Publishing Inc. This work is licensed under the Creative Commons Attribution International License (CC BY 4.0).

http://creativecommons.org/licenses/by/4.0/

\begin{abstract}
The law of mass action, based on maxwellian statistics, cannot explain recent epicatalysis experiments but does when generalized to non-maxwellian statistics. Challenges to the second law are traced to statistical heterogeneity that falls outside assumptions of homogeneity and indistinguishability made by Boltzmann, Gibbs, Tolman and Von Neumann in their H-Theorems. Epicatalysis operates outside these assumptions. Hence, H-Theorems do not apply to it and the second law is bypassed, not broken. There is no contradiction with correctly understood established physics. Other phenomena also based on heterogeneous statistics include non-maxwellian adsorption, the field-induced thermoelectric effect and the reciprocal Hall effect. Elementary particles have well known distributions such as Fermi-Dirac and Bose Einstein, but composite particles such as those involved in chemical reactions, have complex intractable statistics not necessarily maxwellian and best determined by quantum modeling methods. A step by step solution for finding the quantum thermodynamic properties of a quantum composite gas, that avoids the computational requirement of modeling a large number of composite particles includes 1) quantum molecular modeling of a few particles, 2) determining their available microstates, 3) producing their partition function, 4) generating their statistics, and 5) producing the epicatalytic parameter for the generalized law of mass action.
\end{abstract}

\section{Keywords}

Adsorption, Boltzmann, Entropy, Epicatalysis, H-Theorem, Heterogeneous Statistics, Law of Mass Action, Non-Maxwellian, Quantum Thermodynamics, Second Law

Only in statistics can odds be even, but evens not odd

${ }^{1}$ In this paradoxical statement the author brings together three important concepts discussed in this paper: statistics, asymmetry, and heterogeneous or categorically mismatched systems. 


\section{Introduction}

Non-maxwellian statistics describe the behavior of large numbers of particles behaving according to the rules of quantum mechanics. For example, elementary particles such as fermions follow the Fermi-Dirac distribution, and bosons, the Bose-Einstein distribution. However, composite particles have more complex statistics. Bonding can add or subtract accessible microstates. If the temperature is too far below a bond's energy, the degree of freedom corresponding to this bond gets "frozen out" leaving the rest of the system to determine the statistical property of the whole composite. Usually, such systems tend, in the limit, towards the classical Maxwell-Boltzmann distribution, but not always, as shall be discussed in this paper.

Quantum thermodynamics is well-developed for elementary particles, but not for composite particles, except in the limiting case of maxwellian statistics. The chemical properties of a single molecule can be obtained using quantum molecular modeling, but the quantum thermodynamic properties of a molecular gas require quantum molecular modeling of a large number of molecules, which is computationally impractical.

Recent laboratory experiments not fitting some well-established paradigms on catalysts, highlight the need for a better understanding of this branch of thermodynamics.

The current theory asserts that catalysts [1] speed up chemical reactions without being consumed by the reactions and without shifting the equilibrium state of the final product. If the equilibrium state were to depend on the presence or absence of a catalyst, then it would be possible to move the equilibrium back and forth between two states, simply by applying or removing the catalyst. This would violate the second law, since heat energy could then be converted to work. Furthermore, two different equilibrium states would coexist, one near the catalyst, the other away from it, and the chemical system would never reach equilibrium thereby violating detailed balance.

Experimental data challenging the status quo is inherently suspicious and must be thoroughly vetted by the scientific community. After thorough verification, ideally by independent researchers in different labs, the data becomes an invaluable impetus for revising current theories. New and more encompassing visions of nature can then be discovered, showing current understanding to be but a special case embedded in a larger framework.

Now, experiments by two independent researchers contradict the catalyst paradigm. They found that a class of catalysts, epicatalysts, can in fact shift the equilibrium state of a reaction.

In his experiments Sheehan [2] [3] [4] [5] [6] shows that two metals, rhenium and tungsten in the presence of hydrogen gas at about $1950^{\circ} \mathrm{K}$ and low pressure develop a steady state temperature differential of $126^{\circ} \mathrm{C}$ with the hydrogen dissociating preferentially at the rhenium catalyst and recombining at the tungsten catalyst.

In room temperature experiments, Miller [7] observed temperature differenc- 
es of about $0.2 \mathrm{C}$ between catalysts operating on gases with weak hydrogen bonds such as formic acid.

As shall be discussed, the law of mass action, as currently understood is based on maxwellian statistics, and cannot explain epicatalysis. In this paper, the law is generalized to non-maxwellian statistics, thereby providing an explanation.

The following shall be discussed.

1) Law of mass action for maxwellian statistics.

2) Law of mass action for non-maxwellian statistics.

3) Maxwellian catalysts.

4) Non-maxwellian symmetrical catalysts.

5) Non-maxwellian asymmetrical catalysts.

6) Heterogeneous epicatalytic systems.

7) Quantum chemistry of epicatalyst.

\section{Law of Mass Action}

This law states that that the rate of a chemical reaction is directly proportional to the product of the activities or concentrations of the reactants. Figure 1 illustrates chemical equilibrium between state $A$ with molar concentration $X_{A}$ at energy $E_{A}$ and state $B$ with molar concentration $X_{B}$ at energy $E_{B}$.

This paper shall use a version of the law that expresses equilibrium by relating the concentration of the reactants with Gibbs free energy between two states:

$$
\Delta G_{A B}=-R \theta_{T} \ln \left(\frac{X_{A}}{X_{B}}\right)
$$

where $\Delta G_{A B}=E_{A}-E_{B}$ is the Gibbs free energy at constant pressure. (The Helmholtz energy is used for constant volume.)

Please note that in keeping with other recent publications by this author, the symbol $\theta_{T}$, called statistical temperature is an input parameter in a given statistical distribution, and $T$, called kinetic temperature, is proportional to the expectation value of kinetic energy calculated using that distribution. The two are related by

$$
T\left(E_{p}, \theta_{T}\right)=\frac{2}{3 k_{B}} \int_{0}^{\infty} E_{k} f\left(E_{k}, E_{p}, \theta_{T}\right)_{\text {normalized }} \mathrm{d} E_{k}
$$

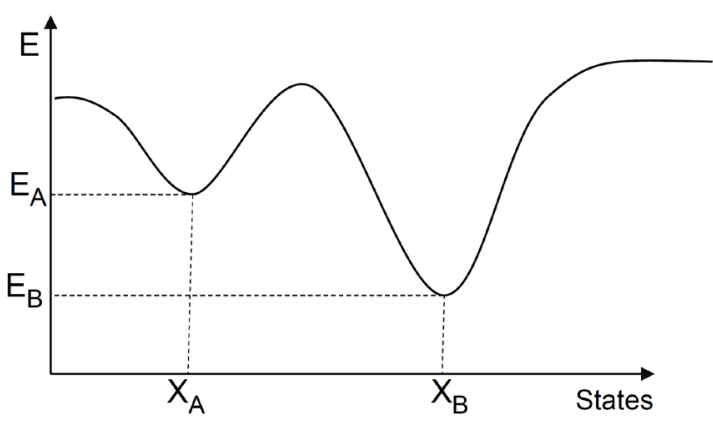

Figure 1. The law of mass action defines the equilibrium between states at different energies. This equilibrium depends on the statistics of particles. 
where $f\left(E_{k}, E_{p}, \theta_{T}\right)$ is the distribution. They are only identical for the Maxwell-Boltzmann distribution [8] [9].

A cursory inspection of the logarithmic/exponential form of Equation (1) suggests that the law of mass action is fundamentally maxwellian. A non-maxwellian version of the law would have to deviate from this form. For example, the Fermi-Dirac distribution includes the term:

$$
\frac{1}{1+\exp \left(\frac{E}{R \theta_{T}}\right)}
$$

which is not strictly exponential. The following two sub-sections scrutinize the foundation of this law which will firstly be derived for maxwellian statistics, and, secondly, be generalized for non-maxwellian statistics.

\subsection{Law of Mass Action for Maxwellian Statistics}

A quick proof of the law of mass action as currently known, will help clarify its basic assumptions. Consider the simple system comprised of a gas in a container. The gas shall be assumed to follow a Maxwell-Boltzmann energy distribution [8] [9] [10]:

$$
f_{M B}\left(E_{k}, \theta_{T}\right)=2\left(\frac{1}{k_{B} \theta_{T}}\right)^{3 / 2} \sqrt{\frac{E_{k}}{\pi}} \exp \left(\frac{-E_{k}}{k_{B} \theta_{T}}\right)
$$

A downward vertical force is applied on the gas. The nature of the force is not important. It can be gravity. It can be an electrical field applied to charged particles. It can also be a Lennard Jones potential. To represent this force, a potential energy term $E_{p}$ (corresponding to elevation $z$ ) is inserted in the exponential term adjacent to the kinetic energy $E_{k}$ :

$$
f_{M B}\left(E_{k}, E_{p}, \theta_{T}\right)=2\left(\frac{1}{k_{B} \theta_{T}}\right)^{3 / 2} \sqrt{\frac{E_{k}}{\pi}} \exp \left(\frac{-E_{k}-E_{p}}{k_{B} \theta_{T}}\right)
$$

The author derived this equation from fundamental principles [9]. The exponential term is the Boltzmann factor. It stands for occupancy per microstate at a given total energy $E_{k}+E_{p}$. The square root term represents the density of states (i.e., the number of microstates per velocity volume in phase space.) This term is proportional to velocity, hence, to the square root of $E_{k}$. The remaining terms, in front, normalize the probability distribution which is occupancy as a function of kinetic energy.

Note that $E_{p}$ is expressed within an exponent, and can be factored out:

$$
f_{M B}\left(E_{k}, E_{p}, \theta_{T}\right)=2\left(\frac{1}{k_{B} \theta_{T}}\right)^{3 / 2} \sqrt{\frac{E_{k}}{\pi}} \exp \left(\frac{-E_{k}}{k_{B} \theta_{T}}\right) \exp \left(\frac{-E_{p}}{k_{B} \theta_{T}}\right)
$$

Combining Equation (4) and Equation (6) yields:

$$
f_{M B}\left(E_{k}, E_{p}, \theta_{T}\right)=f_{M B}\left(E_{k}, E_{p}=0\right) \exp \left(\frac{-E_{p}}{k_{B} \theta_{T}}\right)
$$


Assuming $N_{o}$ particle density at ground level, the lapse in particle density $N_{z} / N_{o}$ at elevation $z$ is:

$$
\frac{N_{z}}{N_{0}}=\frac{\int_{0}^{\infty} f_{M B}\left(E_{k}, E_{p}, \theta_{T}\right) \mathrm{d} E_{k}}{\int_{0}^{\infty} f_{M B}\left(E_{k}, E_{p}=0, \theta_{T}\right) \mathrm{d} E_{k}}
$$

Combining Equation (7) and Equation (8) yields:

$$
\frac{N_{z}}{N_{0}}=\frac{\int_{0}^{\infty} f_{M B}\left(E_{k}, E_{p}=0, \theta_{T}\right) \exp \left(\frac{-E_{p}}{k_{B} \theta_{T}}\right) \mathrm{d} E_{k}}{\int_{0}^{\infty} f_{M B}\left(E_{k}, E_{p}=0, \theta_{T}\right) \mathrm{d} E_{k}}
$$

Hence

$$
\frac{N_{z}}{N_{0}}=\exp \left(\frac{-E_{p}}{k_{B} \theta_{T}}\right)
$$

The equation is the well-known density lapse with elevation in an isothermal atmosphere, which confirms the validity of Equation (5). Equation (10) can also be expressed as:

$$
E_{p}=-k_{B} \theta_{T} \ln \left(\frac{N_{z}}{N_{0}}\right)
$$

If multiple gas species $i=1,2, \cdots$ are considered, one can write

$$
E_{p 1}+E_{p 2}+\cdots=-k_{B} \theta_{T} \ln \left(\frac{N_{z 1} N_{z 2}}{N_{01} N_{02}} \cdots\right)
$$

Expressing all quantities in moles yields:

$$
E_{p 1}+E_{p 2}+\cdots=-R \theta_{T} \ln \left(\frac{C_{z 1} C_{z 2}}{C_{01} C_{02}} \cdots\right)
$$

where $C_{z i}$ is the mole density. This equation leads to the law of mass action for equilibrium between states $A$ and $B$.

$$
\Delta G_{A B}=-R \theta_{T} \ln \left(\frac{X_{A}}{X_{B}}\right)
$$

where $\Delta G_{A B}=E_{p 1}+E_{p 2}+\cdots$ and $X_{A} / X_{B}=K_{e}$, the equilibrium constant.

The proof above is instructive because of its assumptions:

1) The gas is maxwellian (Equation (4)).

2) Implied in assumption 1) is that the gas is statistically homogeneous. Interestingly, Boltzmann assumes gas homogeneity in his proof of the second law-the H-Theorem [11] [12] [13].

The above assumptions suggest that the law of mass action is based on, and must comply with, the second law and cannot support epicatalysis.

\subsection{Law of Mass Action for Non-Maxwellian Statistics}

Consider a gas column comprised of non-maxwellian particles subjected to a force. Let the gas have an arbitrary non-maxwellian energy distribution 
$f\left(E_{k}, E_{p}, \theta_{T}\right)$ which includes a density of state factor and a probability of occupancy factor. For example the distribution of a fermion gas in a potential energy well $E_{p}$ could be expressed as:

$$
f_{\text {Fermions }}\left(E_{k}, E_{p}, \theta_{T}\right)_{\text {normalized }}=A\left(E_{p}, \theta_{T}\right) \sqrt{E_{k}} \frac{1}{1+\exp \left(\frac{E_{k}+E_{c}+E_{p}-E_{F}}{k_{B} \theta_{T}}\right)}
$$

The density of state expressed by the square root term is independent of elevation, not being a function of $E_{p}$. The Fermi-Dirac term, however, is a function of potential energy. As we did in the previous section, the density lapse for a non-maxwellian gas can be written as:

$$
\left(\frac{N_{z}}{N_{0}}\right)_{N o n-M B}=\frac{\int_{0}^{\infty} f\left(E_{k}, E_{p}, \theta_{T}\right) \mathrm{d} E_{k}}{\int_{0}^{\infty} f\left(E_{k}, E_{p}=0, \theta_{T}\right) \mathrm{d} E_{k}}
$$

There is no general analytical solution for the above integral when the distribution $f\left(E_{k}, E_{p}\right)$ is Fermi-Dirac's or Bose-Einstein's, except for specific cases [14], the only method being numerical. Distributions for non-elementary particles such as found in chemical reactions is even more problematic and will be discussed in Section 5 on Quantum Chemistry of Epicatalysts. Given the intractable nature of the integrals, the analytical method used in the previous section cannot be employed here. We shall therefore rely on an ad-hoc approach. Recall that for the Maxwell-Boltzmann distribution:

$$
\left(\frac{N_{z}}{N_{0}}\right)_{M B}=\exp \left(\frac{-E_{p}}{k_{B} \theta_{T}}\right)
$$

Let us define $\alpha$ as the ratio in the density lapse between a non-maxwellian gas and a maxwellian gas:

$$
\alpha=\frac{\left(N_{z} / N_{0}\right)_{N o n-M B}}{\left(N_{z} / N_{0}\right)_{M B}}
$$

and Equation (16) can simply be written as:

$$
\left(N_{z} / N_{0}\right)_{N o n-M B}=\alpha\left(N_{z} / N_{0}\right)_{M B}
$$

A numerical solution of $\alpha\left(E_{p}\right)$ for the Fermi-Dirac distribution is shown in Figure 2.

The ratio $\alpha\left(E_{p}, \theta_{T}\right)$ is plotted as a function of $E_{p}$ for the Fermi-Dirac distribution. It increases with elevation indicating that fermions are less compressible than maxwellian particles. (The Fermi energy was arbitrarily selected to be $2 k_{B} \theta_{T}$ ) This behavior is caused by the Pauli exclusion principle. This graph was generated with the assistance of a publicly available non-maxwellian distribution calculator program [15].

Combining Equation (17) and Equation (18) yields:

$$
E_{p}=-k_{B} \theta_{T} \ln \left(\frac{1}{\alpha} \frac{N_{z}}{N_{0}}\right)_{N o n-M B}
$$




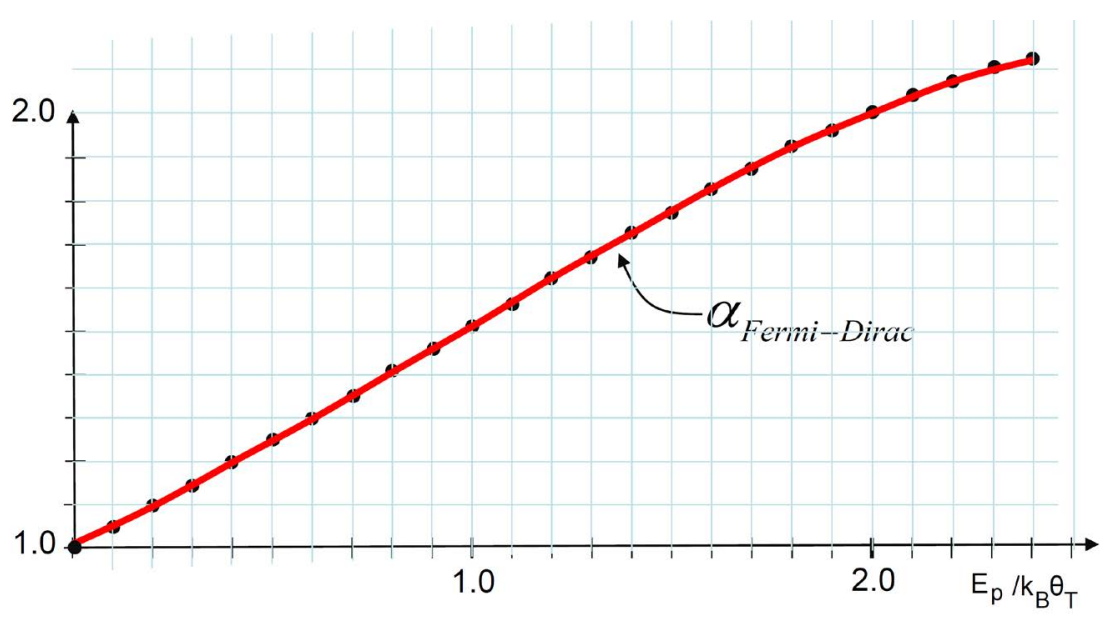

Figure 2. The fermionic/maxwellian density ratio $\alpha$.

If multiple gas species $i=1,2$ are considered, one can write:

$$
E_{p 1}+E_{p 2}+\cdots=-k_{B} \theta_{T} \ln \left(\frac{1}{\alpha_{1} \alpha_{2}} \frac{N_{z 1} N_{z 2}}{N_{01} N_{02}} \cdots\right)_{\text {Non }-M B}
$$

Expressing all quantities in moles, yields:

$$
E_{p 1}+E_{p 2}+\cdots=-R \theta_{T} \ln \left(\frac{1}{\alpha_{1} \alpha_{2}} \frac{C_{z 1} C_{z 2}}{C_{01} C_{02}} \cdots\right)_{\text {Non }-M B}
$$

where $C_{0 i}$ and $C_{z i}$ are mole densities. A state of equilibrium between states $A$ and $B$ for a non-maxwellian gas can be expressed as:

$$
\Delta G_{A B}=-R \theta_{T} \ln \left(\frac{1}{\alpha} \frac{X_{A}}{X_{B}}\right)_{N o n-M B}
$$

where $\Delta G_{12}=E_{p 1}+E_{p 2}+\cdots ; \alpha=\alpha_{1} \alpha_{2} \cdots ;$ and $X_{A} / \alpha X_{B}=K_{e}$, the equilibrium constant.

Equation (23) shows that the equilibrium constant is shifted by the $\alpha$ factor. This equation can alternatively be stated as:

$$
\Delta G_{A B}=-R \theta_{T} \ln \left(\frac{X_{A}}{X_{B}}\right)_{N o n-M B}+R \theta_{T} \ln \alpha
$$

indicating that the Gibbs free energy is shifted by $R \theta_{T} \ln (\alpha)$.

This is the non-maxwellian version of the law of mass action. It suggests that chemical reactions governed by different statistics produce different equilibrium states, and that a static concentration gradient can develop when statistical rules do not apply uniformly between locations.

Does this break the second law? The answer is "not necessarily." A concentration gradient, per se, cannot be used to convert heat to work or to lower entropy, unless it is accessible from the outside. For example, the built-in potential across a diode (charge gradient) cannot be used to generate energy by connecting leads across the diode, because the connections develop their own potentials that ex- 
actly cancel the built-in potential. This idea is developed further below.

\section{Catalysis}

This section covers the operation of catalysts in light of the revised law of mass action. The following are covered:

1) Maxwellian catalysts,

2) Non-maxwellian symmetrical catalyst,

3) Non-maxwellian asymmetrical catalyst,

4) Heterogeneous catalytic systems

\subsection{Maxwellian Catalysts}

As shown in Figure 3, a catalyst facilitates a reaction between two states $A$ and $B$ by introducing an intermediate state $C$ that lowers the energy barrier between $A$ and $B$.

Using the law of mass action of Equation (14), we can express equilibrium between $A$ and $C$ as:

$$
\Delta G_{A C}=-R \theta_{T} \ln \left(\frac{X_{A}}{X_{C}}\right)
$$

and between $C$ and $B$ as:

$$
\Delta G_{C B}=-R \theta_{T} \ln \left(\frac{X_{C}}{X_{B}}\right)
$$

Combining Equation (25) and Equation (26) cancels out the catalytic terms $X_{O}$ leaving:

$$
\Delta G_{A C}+\Delta G_{C B}=-R \theta_{T} \ln \left(\frac{X_{A}}{X_{B}}\right)
$$

Now invoking conservation of energy:

$$
\Delta G_{A B}=\Delta G_{A C}+\Delta G_{C B}
$$

yields:

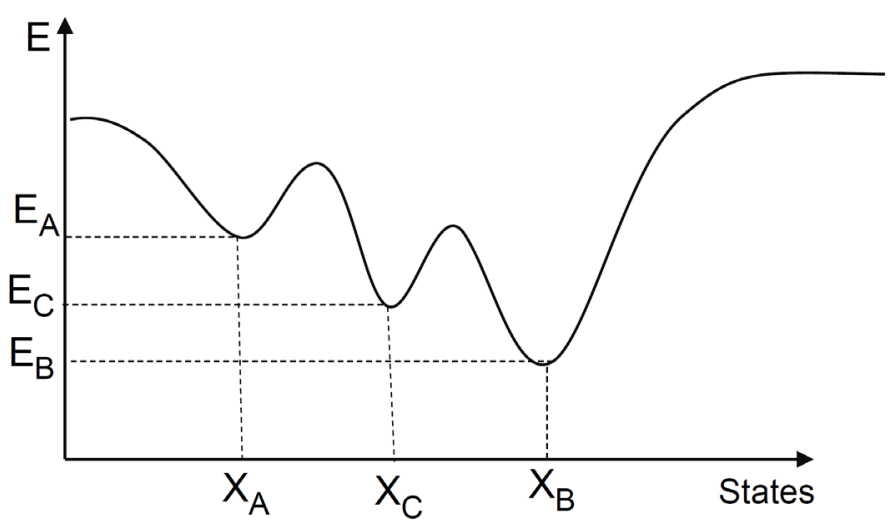

Figure 3. A catalyst creates an intermediate state $C$ that lowers the energy barrier between $A$ and $B$. 


$$
\Delta G_{A B}=-R \theta_{T} \ln \left(\frac{X_{A}}{X_{B}}\right)
$$

This equation is exactly the same equation as the one without the catalyst (14). One must conclude that, in accordance with the established paradigm, a catalyst operating according to a law of mass action based on maxwellian statistics, does not shift the equilibrium state and complies with the second law.

\subsection{Non-Maxwellian Symmetrical Catalysts}

Let us now consider a more complicated system with non-maxwellian statistics as expressed by parameter $\alpha$ in Equation (23). The biasing produced by $\alpha$ shall be assumed to be symmetrical, and all reactants to be equally affected by the catalyst. The interaction between the catalyst and states $A$ and $B$ becomes:

$$
\begin{gathered}
\Delta G_{A C}=-R \theta_{T} \ln \left(\frac{X_{A}}{\alpha X_{C}}\right) \\
\Delta G_{C B}=-R \theta_{T} \ln \left(\frac{\alpha X_{C}}{X_{B}}\right)
\end{gathered}
$$

Combining these two equations cancels out the catalytic terms $\alpha X_{C}$ leaving:

$$
\Delta G_{A C}+\Delta G_{C B}=-R \theta_{T} \ln \left(\frac{X_{A}}{X_{B}}\right)
$$

Now invoking conservation of energy:

$$
\Delta G_{A B}=\Delta G_{A C}+\Delta G_{C B}
$$

yields

$$
\Delta G_{A B}=-R \theta_{T} \ln \left(\frac{X_{A}}{X_{B}}\right)
$$

All terms related to the catalyst have disappeared indicating that the equilibrium has not shifted. Despite being non-maxwellian, a non-maxwellian symmetrical catalyst does not shift the equilibrium state. The catalysis paradigm is preserved, and the second law is not violated.

\subsection{Non-Maxwellian Asymmetrical Catalysts-Epicatalysts}

Let us now consider a catalyst with an asymmetrical statistical relation with reactants. The justification for such a strange chemical is discussed in Section 5 on the quantum chemistry of epicatalysts. The interaction between the catalyst and states $A$ and $B$ becomes:

$$
\begin{gathered}
\Delta G_{A C}=-R \theta_{T} \ln \left(\frac{X_{A}}{\alpha_{A C} X_{C}}\right) \\
\Delta G_{C B}=-R \theta_{T} \ln \left(\frac{\alpha_{B C} X_{C}}{X_{B}}\right)
\end{gathered}
$$

Combining these two equations does not cancel out the $\alpha$ terms: 


$$
\Delta G_{A C}+\Delta G_{C B}=-R \theta_{T} \ln \left(\frac{\alpha_{A C} X_{A}}{\alpha_{B C} X_{B}}\right)
$$

Now invoking conservation of energy:

$$
\Delta G_{A B}=\Delta G_{A C}+\Delta G_{C B}
$$

Therefore:

$$
\Delta G_{A B}=-R \theta_{T} \ln \left(\frac{\alpha_{A C} X_{A}}{\alpha_{B C} X_{B}}\right)
$$

Equivalently,

$$
\Delta G_{A B}=-R \theta_{T} \ln \left(\frac{X_{A}}{X_{B}}\right)+R \theta_{T} \ln \frac{\alpha_{B C}}{\alpha_{A C}}
$$

The equilibrium state is shifted by the statistical asymmetry between the reactants. The catalyst can now be called an epicatalyst. Is the second law violated? It depends. If the reactants and catalyst are in an isolated container, equilibrium is shifted but nothing else happens afterward. Entropy is maximized, ambient heat is not converted to work, perpetual motion does not occur, and the second law is not violated.

This conclusion is consistent with the assumption of homogeneity made by Boltzmann in his H-Theorem. Even though the epicatalysts interact asymmetrically with the different reactants, the epicatalyst itself is homogeneous-there is no spatial heterogeneity. The system must be statistically heterogeneous to be capable of a second law violation. This is the topic of the next section.

\subsection{Heterogeneous Epicatalytic Systems}

Figure 4 illustrates a system comprised of several catalysts, one at least is an epicatalyst.

The system never reaches chemical and thermodynamic equilibrium. On one side of the system an epicatalyst shifts the concentration away from the maxwellian

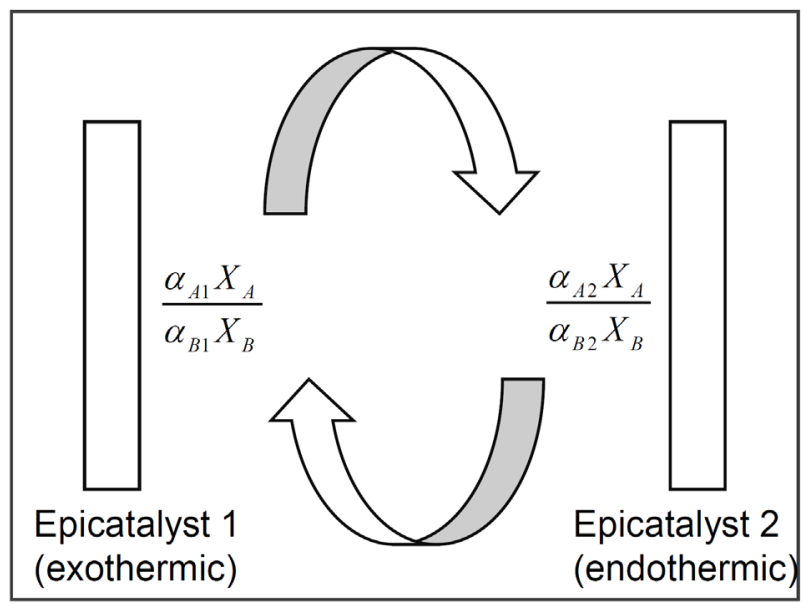

Figure 4. A heterogeneous epicatalytic system closes the loop between an exothermic reaction and an endothermic reaction. 
normal. On the other side, a second catalyst (conventional or epicatalyst) shifts it back. The reaction is exothermic on one side and endothermic on the other side and equilibrium is never reached.

The reaction at the first catalyst can be described by:

$$
\Delta G_{A B}=-R \theta_{T 1} \ln \left(\frac{\alpha_{A C 1} X_{A 1}}{\alpha_{B C 1} X_{B 1}}\right)
$$

and at the second catalyst by:

$$
\Delta G_{A B}=-R \theta_{T 2} \ln \left(\frac{\alpha_{A C 2} X_{A 2}}{\alpha_{B C 2} X_{B 2}}\right)
$$

Since the energy of the reaction is the same on both sides, we can write

$$
\theta_{T 1} \ln \left(\frac{\alpha_{A C 1} X_{A 1}}{\alpha_{B C 1} X_{B 1}}\right)=\theta_{T 2} \ln \left(\frac{\alpha_{A C 2} X_{A 2}}{\alpha_{B C 2} X_{B 2}}\right)
$$

The isothermal case (e.g., applying a thermal short between the catalysts) yields:

$$
\frac{\alpha_{A C 1} X_{A 1}}{\alpha_{B C 1} X_{B 1}}=\frac{\alpha_{A C 2} X_{A 2}}{\alpha_{B C 2} X_{B 2}}
$$

The constant concentration case (e.g., placing the catalysts in two separate containers, thermally insulated from each other, and adjusting the container's temperatures until the concentration ratios are equal) yields:

$$
\theta_{T 1} \ln \left(\frac{\alpha_{A C 1} X_{A}}{\alpha_{B C 1} X_{B}}\right)=\theta_{T 2} \ln \left(\frac{\alpha_{A C 2} X_{A}}{\alpha_{B C 2} X_{B}}\right)
$$

\section{Adsorption}

Epicatalytic effects can also by produced by non-maxwellian adsorption. We shall discuss, firstly, maxwellian adsorption to establish a baseline and secondly, non-maxwellian adsorption.

\subsection{Maxwellian Adsorption}

Adsorption can be modeled as shown in Figure 5.

Consider Equation (7) and let's assume that at ground level the distribution $f_{M B}\left(E_{k}, E_{p}=0, \theta_{T}\right)$ is normalized. At elevation $z$ (or equivalently at potential energy $\left.E_{p}\right)$ the factor $\exp \left(-E_{p} / k_{B} \theta_{T}\right)$ scales down $f_{M B}\left(E_{k}, E_{p}, \theta_{T}\right)$, but also denormalizes it. One can renormalize it simply by dividing it by $\exp \left(-E_{p} / k_{B} \theta_{T}\right)$. After renormalization, the distribution at elevation $z$ is identical to the one at ground level [8] [9]:

$$
f_{M B}\left(E_{k}, E_{p}, \theta_{T}\right)_{\text {normalized }}=f_{M B}\left(E_{k}, E_{p}=0, \theta_{T}\right)_{\text {normalized }}
$$

Since $\theta_{T}$ is a parameter of the distribution that spans the height of the whole column, the gas is isothermal with respect statistical temperature. Furthermore, since the kinetic energy distribution (after renomalization) is independent of $E_{p}$ or $z$ as indicated by Equation (46), the gas is also isothermal with respect kinetic 


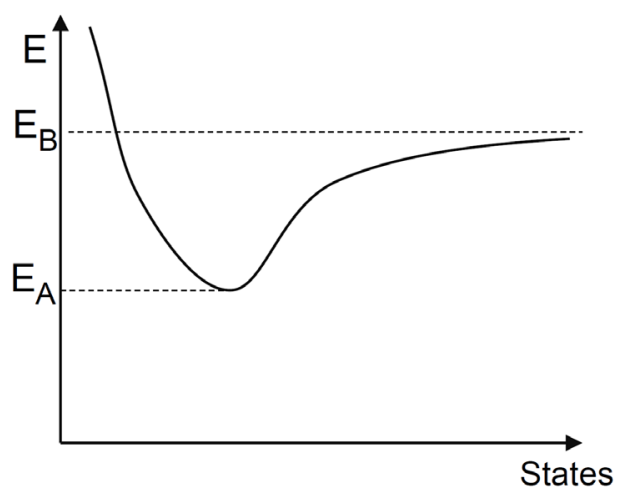

Figure 5. The Lennard-Jones potential can model adsorption.

temperature $T$. Therefore, the gas is in full compliance with the second law as expressed by Clausius (no equilibrium temperature gradient).

Furthermore, the ratio in density for an energy difference $\Delta G=E_{B}-E_{A}$ is given by the mass action Equation (14).

$$
\frac{X_{A}}{X_{B}}=\exp \left(-\frac{E_{A}-E_{B}}{R \theta_{T}}\right)
$$

which is the well-known lapse in density with elevation for an isothermal atmosphere.

\subsection{Non-Maxwellian Adsorption}

When the distribution function is non-maxwellian, renormalization cannot restore it to its form at ground level because potential energy is not expressed exponentially and cannot be factored out. Even though the statistical temperature parameter $\theta_{T}$ is constant with elevation because the statistics is invariant for the ensemble, the gas is not isothermal with respect to the kinetic temperature $T$. Figure 6(a) shows a maxwellian distribution, and Figure 6(b) a Fermi-Dirac distribution, the red thick curve representing ground level and the blue thin curve, elevation $z$. The maxwellian distribution is invariant, but Fermi-Dirac's is shifted to the left indicating a temperature drop with elevation. Therefore, a non-maxwellian adsorber can produce a temperature gradient $\mathrm{d} T / \mathrm{d} z$ in a gas and therefore, an epicatalytic effect [8] [9].

Furthermore the ratio in density is given by Equation (23) which can be expressed for an adsorber as:

$$
\frac{X_{A}}{X_{B}}=\alpha \exp \left(-\frac{E_{A}-E_{B}}{R \theta_{T}}\right)
$$

As already discussed in Section 3 on epicatalysis, a temperature gradient, per se, cannot be used to make a perpetual motion machine unless the system is statistically heterogeneous. In other words, the thermal loop needs to be closed with statistically different thermal carriers. Such systems fall outside of the coverage of the H-Theorem. The second law is not violated, it is bypassed. This topic is extensively discussed by the author in [8] [9] [10] [16] [17] [18]. 


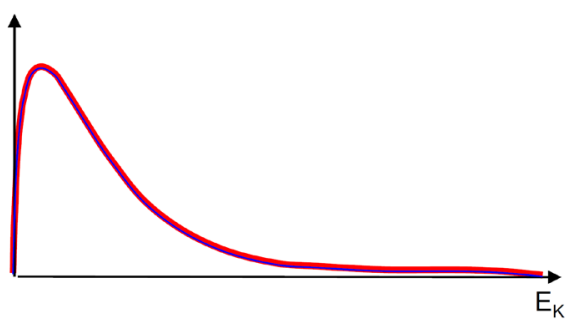

(a)

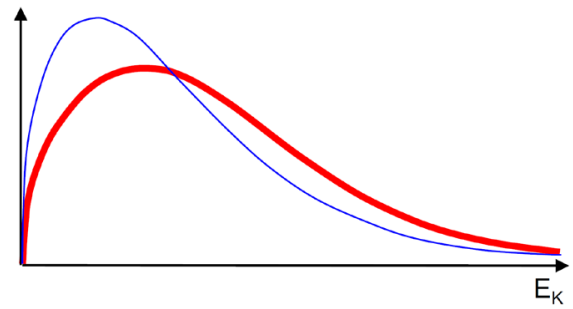

(b)

Figure 6. Normalized distribution represented at ground level by the red thick curve, and at elevation $z$, by the blue thin curve. (a) It is invariant with $z$ when maxwellian and (b) shifted to the left when Fermi-Dirac's [8] [9].

\section{Quantum Chemistry of Epicatalysts}

Epicatalysis occurs when quantum statistics bias the physical/chemical equilibrium between a catalyst and reactants. These statistics are unusual because they describe the behavior of composite systems, not of elementary particles for which the Fermi-Dirac or Bose-Einstein distributions would be adequate. Deviations from these elementary distributions are well known, particularly with regards the statistics of donor and acceptor dopants in semiconductors [19] [20]. An electron provided by a donor has a degeneracy of 2 resulting in the distribution:

$$
F_{D}(E)=\frac{1}{1+\frac{1}{2} \exp \left(\frac{E_{D}-E_{F}}{k_{B} \theta_{T}}\right)}
$$

Similarly, the distribution for an acceptor is:

$$
F_{A}(E)=\frac{1}{1+4 \exp \left(\frac{E_{A}-E_{F}}{k_{B} \theta_{T}}\right)}
$$

The statistics of composite particles is more complex than that of elementary particles. Bonding can add or subtract accessible microstates. If the temperature is too far below a bond's energy, the degree of freedom corresponding to this bond gets "frozen out" leaving the rest of the system to determine the statistical property of the whole composite. For example, a hydrogen atom behaves as a boson so long as the temperature is below that required for dissociation. At low enough temperature, two electrons in a solid Cooper pair, can form a single boson, macroscopically observed as superconductivity.

Consider a chemical reaction in which a mixture of $H$ atoms and $H_{2}$ molecules are adsorbed onto an epicatalytic surface. Depending on the chemical interaction with the surface, and the type of bonds formed, electrons could be exchanged in various degrees resulting in $H$ becoming a (partial) donor and $H_{2}$ a (partial) acceptor, or vice versa, thereby giving rise to the statistical asymmetry required by an epicatalyst. A precise answer to this question requires quantum molecular modeling of the catalyst and the reactants. Sheehan's experiments [2] 
[3] [4] [5] [6] utilize tungsten and rhenium as catalysts operating on hydrogen at high temperature. At least one of these two metals is an epicatalyst. It would be instructive to replace these metals, one at a time with a known conventional catalyst to isolate which one has epicatalytic behavior.

The theoretical determination of which metal and which gas can support epicatalytic behavior is beyond the scope of this paper. Given the complexity of the statistics for composite particles, a general analytical solution is probably impossible. For a detailed explanation of epicatalysis, experiments and numerical approaches such as quantum molecular modeling are the only viable alternatives.

\section{Current Research on Statistically Heterogeneous Systems}

This section discusses two other effects which, like epicatalysis, rely on statistical heterogeneous systems to bypass the second law.

\subsection{Field-Induced Thermoelectric Effect}

Experiments on thermoelectric materials produced unexplained data. Despite very meticulous laboratory procedures in the measurement of the Seebeck coefficient, the voltage/temperature curve did not go through the origin indicating a voltage output without a temperature difference input and a temperature difference output without a voltage input, apparently contradicting the second law [8] [10] [16] [21] as shown in Figure 7.

Levy [8] [10] [16] explains this phenomenon as a spontaneous temperature difference in the thermoelectric junction generated multiple statistics-the fermion statistics of the electrical carriers and the boson statistics of the thermal phonons. This temperature gradient is not ordinarily observable in a conventional semiconductor junction because it is shorted by the highly conductive heat phonons. However, it can be measured in high performance thermoelectrics

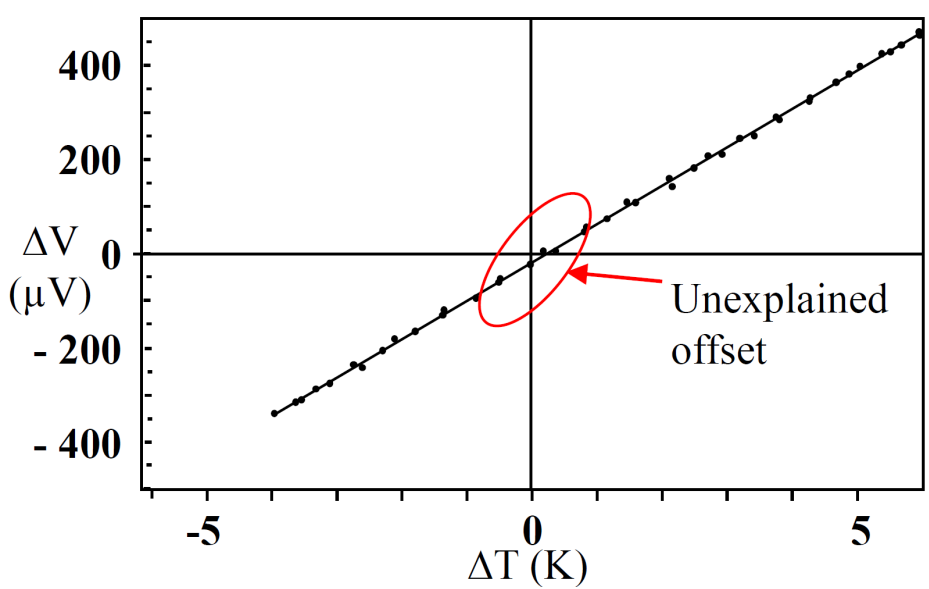

Figure 7. The Seebeck curve does not go through the origin indicating a voltage output without a temperature difference input and a temperature difference output without a voltage input, in apparent violation of the second law [21]. 
in which phonons have a much smaller conductivity. This phenomenon is a field-induced temperature gradient, different from conventional thermoelectric effects, in particular, Peltier's which is a current-induced temperature gradient. It is also different from the reciprocal Hall effect, a field-induced current, which is covered in the next section.

\subsection{Reciprocal Hall Effect}

In the presence of a magnetic field parallel to a surface, and an electric field perpendicular to the surface, and when the surface is in contact with a gas of charged particles, a current is generated in the gas along the surface, perpendicular to both fields [22] as shown in Figure 8.

The configuration of the fields and the surface is shown in Figure $8(a)$. The velocity distribution is shown in Figure $8(\mathrm{~b})$.

The gas acquires a half-maxwellian velocity distribution from the surface. In the absence of field, this distribution is symmetrical around the normal to the surface as shown by the thin blue curve in Figure 8(b), Figure 8(c). The magnetic and electric fields skew the distribution away from the normal as shown by the thick red curves, giving rise to a current in apparent violation of the second law. The horizontal velocity distribution of electrons is shown in red at elevation (b) $200 \mathrm{~nm}$ and (c) $400 \mathrm{~nm}$ above the surface for a magnetic field of 0.2 Tesla. These distributions were generated by a publicly available Reciprocal Hall Effect calculator program [23].

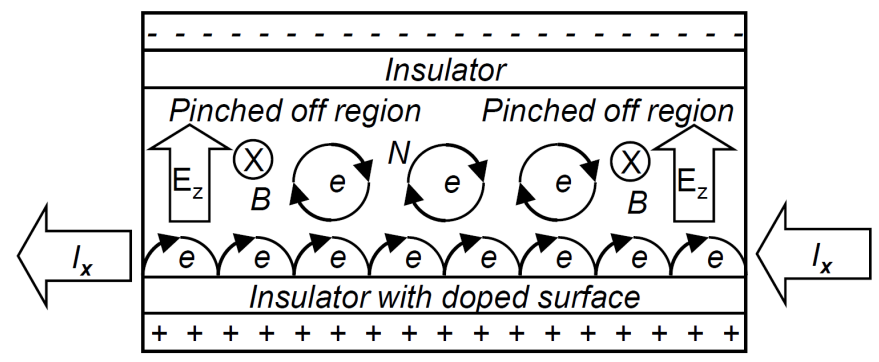

(a)

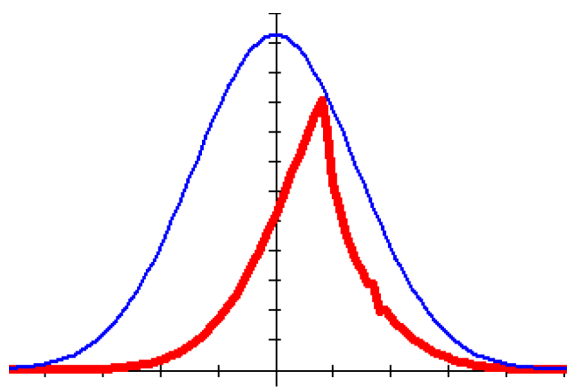

(b)

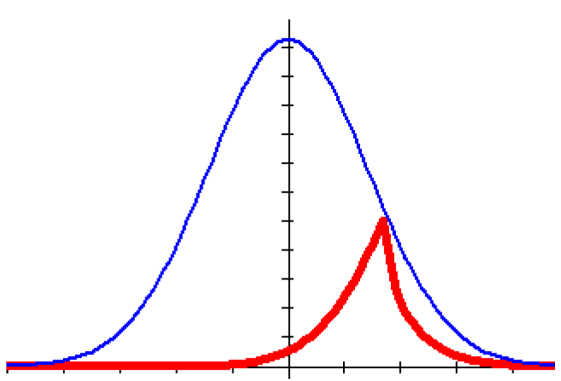

(c)

Figure 8. Reciprocal Hall Effect. The horizontal velocity distribution of electrons is shown in red at elevation (b) $200 \mathrm{~nm}$ and (c) $400 \mathrm{~nm}$ above the surface for a magnetic field of 0.2 Tesla. The thin blue curve provides a reference showing the distribution at zero elevation. The horizontal axis shows $4039 \mathrm{~m} / \mathrm{sec}$ per division. 


\section{Suggested Future Research}

The hypothesis presented in this paper explains epicatalysis by requiring particles involved in the reaction to possess non-maxwellian quantum statistics. Generally, these particles are composite. Therefore, the Fermi-Dirac and Bose-Einstein distributions would be inadequate. A full thermodynamic analysis would require a quantum molecular simulation of large numbers of atoms and molecules, clearly beyond the capability of current computers.

However, this problem is not unsolvable. The following steps are suggested:

1) Quantum molecular modeling of the interaction between few particles. For example, a hydrogen molecule in contact with a tungsten surface. This is clearly within current computer capabilities.

2) Evaluating the number of microstates that can be achieved in this interaction, and evaluation of the partition function.

3) Determining molecular statistics (for a much larger number of molecules) using the partition function.

4) From the molecular statistics, evaluating the epicatalytic parameter $\alpha$ from which the corresponding non-maxwellian law of mass action can be derived, as described in this article. The analytical tools provided in this paper can then be used to evaluate the performance of the epicatalyst.

Other experimental data that also challenge the second law. Motley [24] and Rynn [25] describe epicatalytic behavior in Q machines (plasma devices). Linking these phenomena with the presence of heterogeneous non-maxwellian statistics could additionally be a topic of future research.

\section{Conclusions}

Unlike other branches of physics, thermodynamics has barely evolved since its founding days in the nineteenth century. In his H-Theorem, Boltzmann proves that entropy never decreases in an isolated gas. However, he crucially assumes that the gas is homogeneously distributed [11] [12] [13]. Gibbs makes the further assumption that the gas particles are indistinguishable [26]. (He discusses several types of heterogeneous systems in [27] but does not cover statistically heterogeneous systems.) These assumptions by Boltzmann and Gibbs imply statistical homogeneity/indistinguishability. In those days (late nineteenth century), gases were only known to follow the classical Maxwell-Boltzmann distribution and Boltzmann and Gibbs assumptions were not and could not have been regarded as over-restrictive given the limitations of classical physics of the time. Quantum statistics were discovered in the early twentieth century. Yet Tolman and Von Neumann who developed their own H-Theorem for quantum gases [28] [29] [30], also had to assume homogeneity/indistinguishability. Unfortunately, none of these researchers looked at implications of heterogeneous systems containing macroscopically distinguishable components on the basis of their statistics (i.e., fermions vs. bosons).

Does a heterogeneous epicatalytic system break the second law? It all depends 
how one defines the law. As stated by Boltzmann, Tolman and Von Neumann (entropy never decreases) it is only valid within a restricted domain limited by homogeneity/indistinguishability assumptions of their H-Theorems. Heterogenous statistical systems bypass the law simply because they fall outside of these assumptions. The law is not broken, it is bypassed. The hypothesis proposed in this paper is consistent with correctly understood established physics. There is no contradiction. Are the $\mathrm{H}$-Theorems flawed because of their limitations? Not at all. However, expecting the law to be universal is flawed.

Other versions of the law [31], for example, heat flows from hot to cold, (Clausius,) and perpetual motion machines are impossible, (Kelvin-Planck,) have the weakness of having been inductively/empirically derived. Induction is a poor guide as illustrated in Bertrand Russell/farmer/chicken paradox. The chicken being fed daily by the farmer, thought it was safe, until one day the farmer wrung its neck.

Sheehan's epicatalysis experiments strikingly invalidate the inductively derived versions of the law. His data provided the author with the impetus to scrutinize the foundations of catalysis. The law of mass action was generalized to cover non-maxwellian systems. Epicatalysts were found capable of bypassing the second law but only when placed in a heterogeneous statistical system comprised of multiple reaction sites operating according to different statistics. In other words, the chemical loop must be closed. Other promising approaches using heterogeneous statistics includes non-maxwellian adsorption, the field-induced thermoelectric effect and the reciprocal hall effect.

Experimental data not fitting current theories are precious. They offer a rare opportunity to extend science and take a step towards clean and inexpensive energy and a more environmentally friendly tomorrow.

\section{Acknowledgements}

I would like to thank my wife Penny for her unwavering support.

\section{Conflicts of Interest}

The author declares no conflicts of interest regarding the publication of this paper.

\section{References}

[1] Wikipedia, Catalysis. https://en.wikipedia.org/wiki/Catalysis

[2] Sheehan, D.P. (2001) The Second Law and Chemically-Induced, Steady-State Pressure Gradients: Controversy, Corroboration and Caveats. Physics Letters A, 280, 185-190.

[3] Sheehan, D.P., Garamella, J.T., Mallin, D.J. and Sheehan, W.F. (2012) Steady-State Non-Equilibrium Temperature Gradients in Hydrogen Gas-Metal Systems: Challenging the Second Law of Thermodynamics. The Royal Swedish Academy of Sciences, Physica Scripta, Volume 2012, 2012 T51, 014030.

https://inis.iaea.org/search/search.aspx?orig_q=RN:44040484 
[4] Sheehan, D.P. (2013) Nonequilibrium Heterogenous Catalysis in Long Term Mean-Free-Path Regime. Physical Review E, 88, 032125. https://doi.org/10.1103/PhysRevE.88.032125

[5] Sheehan, D.P., Mallin, D.J., Garamella, J.T. and Sheehan, W.F. (2014) Experimental Test of a Thermodynamic Paradox. Foundations of Physics, 44, 235-247. https://doi.org/10.1007/s10701-014-9781-5

[6] Sheehan, D.P., Zawlacki, T.A. and Helmer, W.H. (2016) Apparatus for Testing Gas-Surface Reactions for Epicatalysis. Review of Scientific Instruments, 87, 074101. https://doi.org/10.1063/1.4954971

[7] Miller, D.W. (2016) Apparatus and Procedures for Detecting Temperature Differentials with Epicatalytic Materials, AAAS Pacific Division, 2016 San Diego Meeting, Symposium Abstracts, Limits to the Second Law of Thermodynamics. http://associations.sou.edu/aaaspd/2016SANDIEGO/2016SympAbstracts/12.pdf

[8] Levy, G.S. (2018) Temperature and Voltage Offsets in High ZT Thermoelectrics. Journal of Electronic Materials, 47, 3067-3076.

[9] Levy, G.S. (2015) Quantum Game Beats Classical Odds-Thermodynamics Implications. Entropy, 17, 7645-7657. https://doi.org/10.3390/e17117645

[10] Levy, G.S. (2013) Thermoelectric Effects under Adiabatic Conditions. Entropy, 15, 4700-4715. https://doi.org/10.3390/e15114700

[11] Boltzmann, L. (1872) Weitere Studien über das Wärmegleichgewicht unter Gasmolekülen. In: Sitzungsberichte Akademie der Wissenschaften, Vol. 66, 275-370. (English translation: Boltzmann, L. (2003) Further Studies on the Thermal Equilibrium of Gas Molecules. The Kinetic Theory of Gases. History of Modern Physical Sciences, Vol. 1, 262-349.) https://doi.org/10.1142/9781848161337_0015

[12] Uffink, J. (2006) Compendium of the Foundations of Classical Statistical Physics. 44, 45. http://philsci-archive.pitt.edu/2691/1/UffinkFinal.pdf

[13] Brown, H. and Myrvold, W. (2008) PhilSci-Archive. 09/2008. http://www.socsci.uci.edu/ bskyrms/bio/readings/brown_myrvold_on_boltzmann. pdf http://philsci-archive.pitt.edu/4187/1/Reversibility08.pdf

[14] Kim, R. and Lundstrom, M. Note on Fermi Integrals. arXiv:0811.0116. https://arxiv.org/abs/0811.0116

[15] Levy, G.S. (2017) Non-Maxwellian Distribution Calculator. http://www.entropicpower.com

[16] Levy, G.S. (2016) Anomalous Temperature Gradient in Non-Maxwellian Gases. Proceedings of the 11 th International Conference on Ceramic Materials \& Components for Energy \& Environmental Applications, Vancouver, 14-19 June 2015, Volume 255 .

[17] Levy, G.S. (2017) Playing Rock, Paper, Scissors in Non-Transitive Statistical Thermodynamics. Journal of Applied Mathematics and Physics, 5, 1174-1197.

[18] Levy, G.S. (2018) Using Quantum Statistics to Win at Thermodynamics, and Cheating in Vegas. Journal of Applied Mathematics and Physics, 6, 2166-2179. https://doi.org/10.4236/jamp.2018.610182

[19] Van Zeghbroek, B. (2018) Principles of Semiconductor Devices. Section 2.5.3. http://ecee.colorado.edu/ bart/book/book/

[20] Grundmann, M. (2006) The Physics of Semiconductors. Springer, Berlin Heidelberg, New York, Section 7.5. 
http://www.fulviofrisone.com/attachments/article/403/The Physics of Semiconductors.pdf

[21] Iwanaga, S., Toberer, E.S., LaLonde, A. and Snyder, G.J. (2011) A High Temperature Apparatus for Measurement of the Seebeck Coefficient. Review of Scientific Instruments, 82, Article ID: 063905.

[22] Levy, G.S. (2017) Choosing between the Reciprocal Hall Effect, CPT Symmetry and the Second Law. The Open Science Journal of Modern Physics, 4, 1-8.

https://www.researchgate.net/publication/314899020

[23] Levy, G.S. (2017) Reciprocal Hall Effect Calculator. http://www.entropicpower.com

[24] Motley, R.W. (1975) Q-Machines. Academic Press, New York. https://doi.org/10.1016/B978-0-12-508650-9.50006-0

[25] Rynn, N. and D’Angelo, N. (1960) Device for Generating a Low Temperature, Highly Ionized Cesium Plasma. Review of Scientific Instruments, 31, 1326-1333. https://doi.org/10.1063/1.1716884

[26] Gibbs Paradox. Wikipedia. https://en.wikipedia.org/wiki/Gibbs_paradox

[27] Gibbs, J.W. (1878) On the Equilibrium of Heterogeneous Substances. American Journal of Science, 16, 441-458.

https://radarmet.atmos.colostate.edu/AT620/papers/Gibbs1875-1878-Equilibrium _ of_Heterogeneous_Substances.pdf

[28] Tolman, R.C. (1938) The Principle of Statistical Mechanics. Clarendon Press, Oxford.

https://archive.org/details/ThePrinciplesOfStatisticalMechanicsTolmanOxfordAtTh eClarendonPress 1938

[29] Von Neumann, J. (2010) Proof of the Ergodic Theorem and the H-Theorem in Quantum Mechanics 10/5/1929. Translator: Roderich Tumulka.

[30] Von Neumann, J. (1955) Mathematical Foundations of Quantum Mechanics. Princeton University Press, Princeton.

[31] Capeck, V. and Sheehan, D. (2005) Challenges to the Second Law of Thermodynamics. Springer, Berlin. https://doi.org/10.1007/1-4020-3016-9 